\title{
Editorial
}

\section{Production, Characterization, and Applications of Porous Materials}

\author{
Nikolaos Michailidis, ${ }^{1}$ Alexander Tsouknidas, ${ }^{2}$ Louis-Philippe Lefebvre, ${ }^{3}$ \\ Thomas Hipke, ${ }^{4}$ and Naoyuki Kanetake ${ }^{5}$ \\ ${ }^{1}$ Physical Metallurgy Laboratory, Mechanical Engineering Department, Aristotle University of Thessaloniki, \\ P.O. Box 490, 54124 Thessaloniki, Greece \\ ${ }^{2}$ Department of Mechanical Engineering, Frederick University, 1036 Nicosia, Cyprus \\ ${ }^{3}$ National Research Council Canada, 75 de Mortagne, Boucherville, QC, Canada H1Y 2 V9 \\ ${ }^{4}$ Department of Light Weight Design, Metal Foam Centre, Fraunhofer Institute for Machine Tools and Forming Technologies, \\ 09126 Chemnitz, Germany \\ ${ }^{5}$ Department of Materials Science and Engineering, Graduate School of Engineering, Nagoya University, Furo-cho, \\ Chikusa-ku, Nagoya 464-8603, Japan \\ Correspondence should be addressed to Nikolaos Michailidis; nmichail@eng.auth.gr
}

Received 6 July 2014; Accepted 6 July 2014; Published 12 August 2014

Copyright (C) 2014 Nikolaos Michailidis et al. This is an open access article distributed under the Creative Commons Attribution License, which permits unrestricted use, distribution, and reproduction in any medium, provided the original work is properly cited.

\section{Introduction}

Lightening a structure through the use of holes is a rather ancient concept that naturally led to the development of the new porous structures we are seeing today. A pore within a solid, or cell, can be defined as a spatial confinement of air. The shape and size of the cell have significant influence on the response of the material to external stimuli. The ability to describe the behavior of these materials has enabled engineers to tailor the macromechanical properties of porous materials to specific requirements. This has considerably promoted the applicability of such structures, which are playing an increasingly important role in several high impact sectors (aerospace, automotive, medical devices, etc.). This can be attributed to their unique combination of properties such as high specific mechanical properties, permeability, specific surface area, and biomimetic properties. Recent advances in material synthesis have helped in adapting the properties of porous structures to specific applications. The technical challenge to comprehend and predict the response of these materials when subjected to load has attracted the interest of engineers and materials scientists. However, porous materials exhibit other characteristics of enormous practical importance and thus the capacity to systematically and effectively describe their performance in a specific environment, is of great importance to multiple scientific fields.

The macromechanical response of a noncontinuous medium under load is nonetheless a multidisciplinary phenomenon of simultaneously occurring mechanical and physical processes. The continuous modifications of the structures during deformation add further complexity to predicting their elastic and plastic behavior.

Finite element techniques have helped to ease this and related modeling techniques are now considered as ubiquitous in describing the behavior of porous materials. Advanced mesh generating techniques combined with a recent increase in computational efficiency of processing software have facilitated the adaptation of such methodologies to stochastic geometries as ceramic and metallic foams [1].

\section{Current Trends}

Recent developments in the field of porous materials have helped the commercialization of new porous materials. Cost efficient production processes enabled their acceptance in several industrial sectors.

Open and closed cell structures with a stochastic macroporosity are currently produced with space holder based 
techniques, gas expansion methods (through the addition of a decomposing foaming agent) or even chemical reaction techniques $[2,3]$. These approaches do, however, allow the control of pore size and distribution, to an adequate extent.

Additive manufacturing techniques permit the integration of oriented patterns of pores in a controlled manner. Layer based fabrication techniques have gained tremendous importance in the production of a variety of materials with designed porosity (i.e., metallic, polymer, and composites). A significant limitation of most commercially available printing devices is, however, associated with high material and maintenance costs. Open source rapid prototyping has recently emerged as a cost efficient alternative and may help spreading the use of these technologies, even for composite materials [4].

Several techniques have also been introduced for the production of micro- and mesoporous media, such as carbonization methods, imprinting of nanopores in spin-on dielectric thin films, and hard template methods.

\section{Emerging Applications}

The continuing efforts to explore the interdisciplinary phenomena governing porous materials are dictated by a rapid growth of technology driven sectors such as medicine, pharmaceuticals, and energy, as recent breakthroughs in mesoand macroporous structures have fostered unprecedented opportunities for new applications $[5,6]$. Materials with mesoporosities ranging of $2-50 \mathrm{~nm}$ have been used in drug delivery systems whereas macroporous bioactive ceramics loaded with stem cells and molecules are presently investigated to increase tissue regeneration in large osseous defects $[5,7]$. Metallic-organic frameworks, employed as catalysts or energy conversion and storage devices, molecular sieves and markers, optics and photonics, inverse opals, and so forth, are also among the numerous novel applications of porous materials being developed.

\section{Epilogue}

Our sincere hope and wish is that the recent development presented in this issue will contribute to the development of this multidisciplinary discipline and provide tools to better understand the properties and characteristics of these materials.

Nikolaos Michailidis
Alexander Tsouknidas
Louis-Philippe Lefebvre
Thomas Hipke
Naoyuki Kanetake

\section{References}

[1] N. Michailidis, E. Smyrnaios, G. Maliaris, F. Stergioudi, and A. Tsouknidas, "Mechanical response and FEM modeling of porous $\mathrm{Al}$ under static and dynamic loads," Advanced Engineering Materials, vol. 16, pp. 289-294, 2013.
[2] N. Michailidis and F. Stergioudi, "Establishment of process parameters for producing Al-foam by dissolution and powder sintering method," Materials and Design, vol. 32, no. 3, pp. 15591564, 2011.

[3] N. Kanetake and M. Kobashi, "Innovative processing of porous and cellular materials by chemical reaction," Scripta Materialia, vol. 54, no. 4, pp. 521-525, 2006.

[4] A. Tsouknidas, "Friction induced wear of rapid prototyping generated materials: a review," Advances in Tribology, vol. 2011, Article ID 746270, 7 pages, 2011.

[5] E. Baril, L. P. Lefebvre, and S. A. Hacking, "Direct visualization and quantification of bone growth into porous titanium implants using micro computed tomography," Journal of Materials Science: Materials in Medicine, vol. 22, no. 5, pp. 1321-1332, 2011.

[6] R. Neugebauer and T. Hipke, "Dynamisches verhalten geschäumter bauteile," Materialwissenschaft und Werkstofftechnik, vol. 31, no. 6, pp. 515-518, 2000.

[7] N. Michailidis, F. Stergioudi, K. Viglaki, and M. Chatzinikolaidou, "Production of novel ceramic porous surfaces tailored for bone tissue engineering," CIRP Annals-Manufacturing Technology, vol. 63, no. 1, pp. 557-560, 2014. 

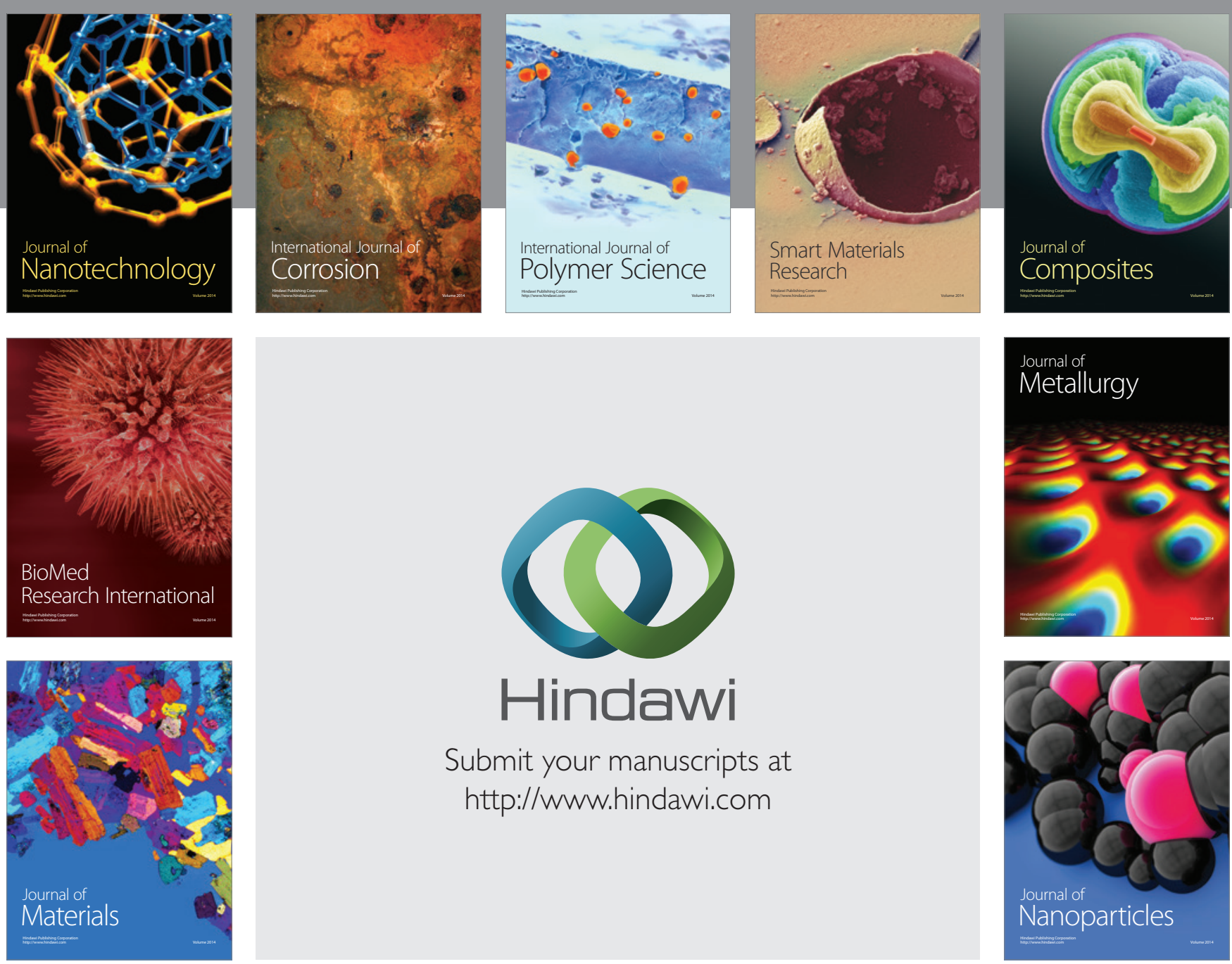

Submit your manuscripts at http://www.hindawi.com
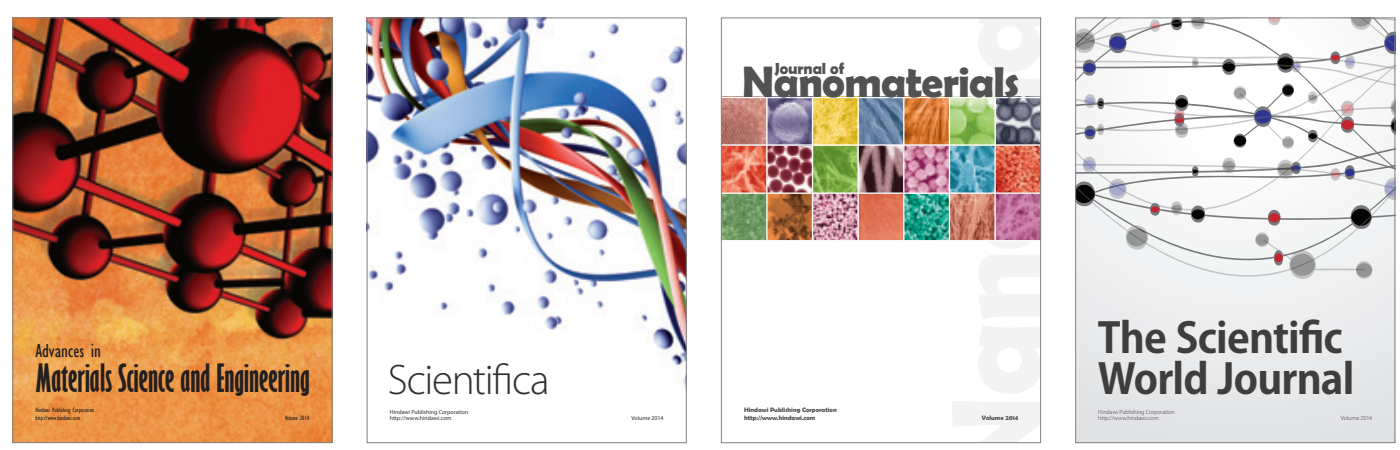

\section{The Scientific World Journal}
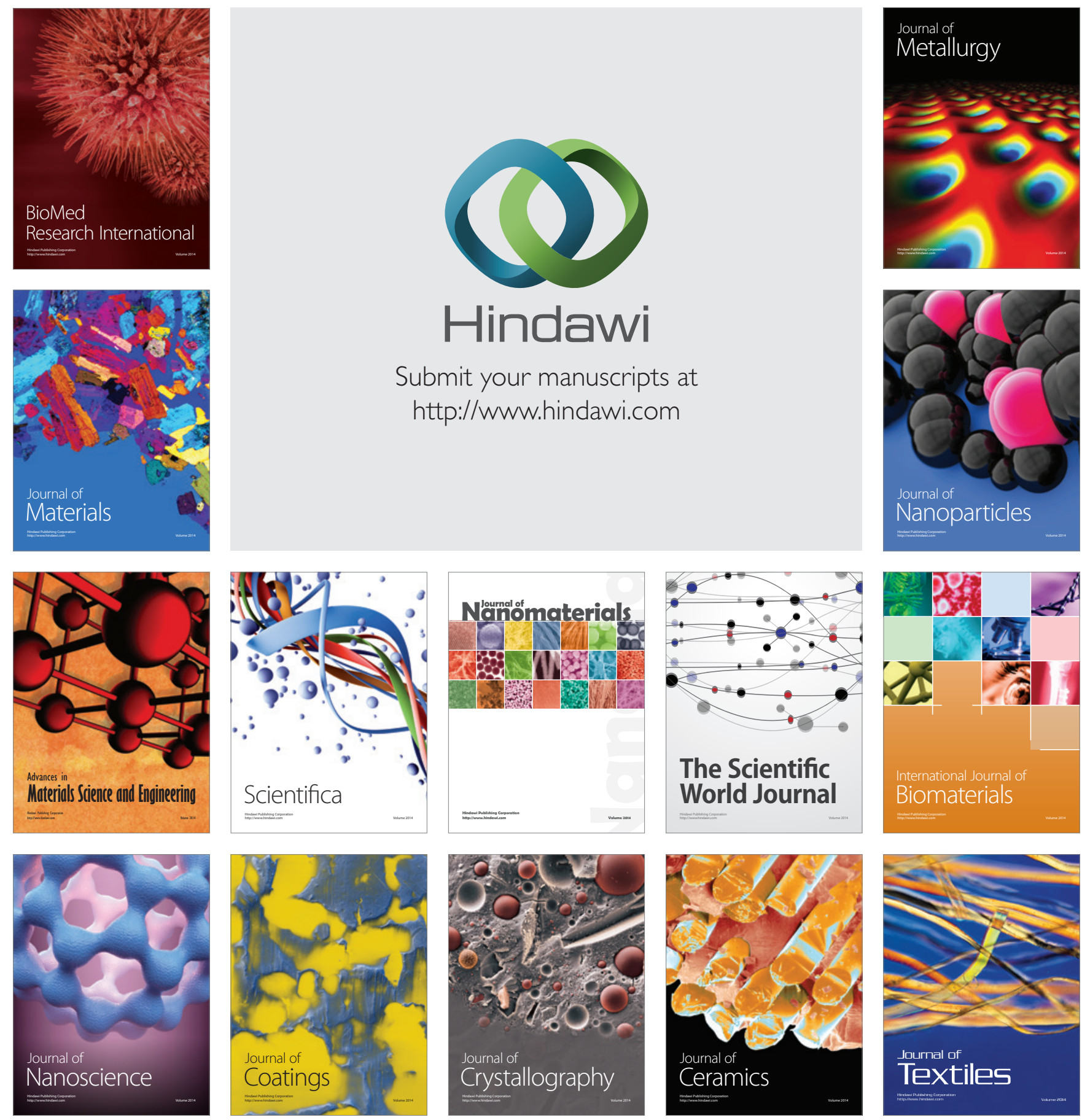